\title{
Optical properties of fully conjugated cyclo[n]thiophenes - An experimental and theoretical approach
}

\author{
Elena Mena-Osteritz ${ }^{1}$, Fan Zhang ${ }^{1}$, Günther Götz ${ }^{1}$, Peter Reineker ${ }^{2}$ \\ and Peter Bäuerle*1
}

\section{Full Research Paper}

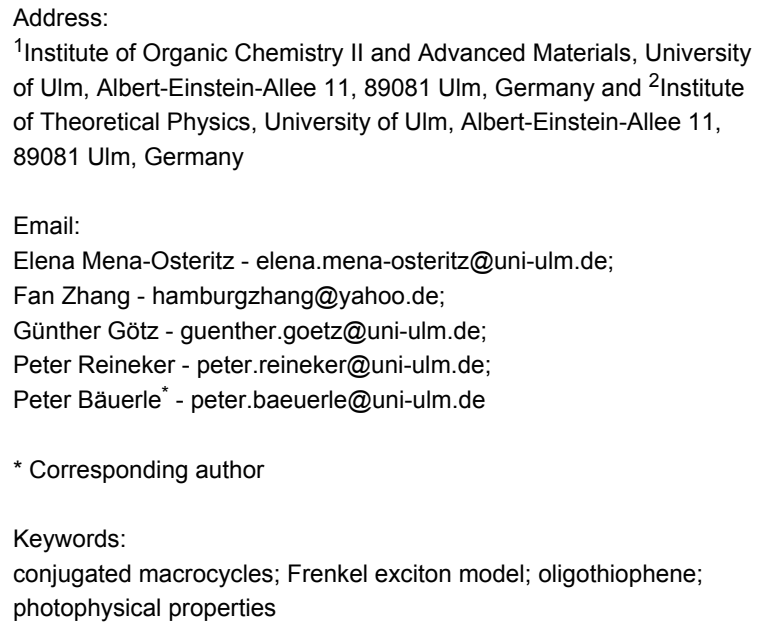

${ }^{1}$ Institute of Organic Chemistry II and Advanced Materials, University of Ulm, Albert-Einstein-Allee 11, 89081 Ulm, Germany and ${ }^{2}$ Institute of Theoretical Physics, University of Ulm, Albert-Einstein-Allee 11, 89081 Ulm, Germany

Email:

Elena Mena-Osteritz - elena.mena-osteritz@uni-ulm.de;

Fan Zhang - hamburgzhang@yahoo.de;

Günther Götz - guenther.goetz@uni-ulm.de;

Peter Reineker - peter.reineker@uni-ulm.de;

Peter Bäuerle* - peter.baeuerle@uni-ulm.de

* Corresponding author

Keywords:

conjugated macrocycles; Frenkel exciton model; oligothiophene;

photophysical properties

Beilstein J. Nanotechnol. 2011, 2, 720-726.

doi:10.3762/bjnano.2.78

Received: 06 July 2011

Accepted: 30 September 2011

Published: 25 October 2011

This article is part of the Thematic Series "Organic-inorganic nanosystems".

Guest Editor: P. Ziemann

(C) 2011 Mena-Osteritz et al; licensee Beilstein-Institut.

License and terms: see end of document.

\begin{abstract}
Optical properties of two series of fully conjugated cyclo[n]thiophenes were analyzed experimentally and theoretically. The absorption spectra reveal a shift to higher wavelengths with increasing size of the cycles, which can be successfully described by an excitonic approach based on a Frenkel exciton Hamiltonian. Furthermore, intriguing new bands in the absorption and fluorescence spectra of the smaller macrocycles disclose the dominance of their ring strain.
\end{abstract}

\section{Introduction}

In the last few decades organic conjugated polymers and oligomers, in particular poly- and oligothiophenes, have attracted a broad interest due to their excellent electronic and transport properties in the solid state, which allow their application in a variety of organic-electronic devices, such as organic field-effect transistors, organic solar cells, and sensors [1-4]. Typically, such $\pi$-conjugated systems comprise extended linear one-dimensional (1D) structures showing interesting optoelectronic properties. In the solid state they represent organic semiconductors, whereas by doping with oxidants metallic states with higher conductivities can be achieved. For various series of 1D linear oligothiophenes, which, in contrast to their polydisperse polymeric counterparts, exhibit defined molecular structures, it was proven that the physical properties correlate well 
with the length of the conjugated chain [5-8]. However, in particular for the shorter oligomers, end-effects imposed by the end groups perturb structure-property correlations, whereas for longer derivatives saturation of, e.g., optical transitions occurs leading to a limiting value. In this respect, 2D macrocyclic systems, cyclo[n]thiophenes $(\mathrm{C} n \mathrm{~T})$ [9-11], which are shapepersistent and cyclically conjugated, were recently introduced by our group. They are not only theoretically most-interesting systems [12-16] providing an infinite $\pi$-conjugated chain like an idealized polymer, but they also represent a novel class of organic semiconductors without end-effects exhibiting fascinating optical [17-20] and self-assembling properties [21-23].

In a statistical macrocyclization approach under high-dilution conditions, the first cyclic representatives C12T, C16T, and C18T were prepared starting from terminally ethynylated terthiophenes, in only low yields and quantities, which is a general observation for the statistical synthesis of macrocycles [24-26]. Somewhat later, we developed a novel method using the same ethynylated oligothiophenes and Pt(II)-precursors as templates leading to stable coordinatively bound metallomacrocycles, which were transformed to the corresponding diacetylene-bridged macrocycles by elimination of the metal centers and simultaneous $\mathrm{C}-\mathrm{C}$ bond formation. In a final step, the diacetylene units were subsequently transformed to thiophene units forming the final cyclothiophene. By this "metal-template approach", among other derivatives the series was extended to C8T as the smallest member and the overall yield was improved to around $10 \%[6,7]$. More recently, by applying $\mathrm{Pt}(\mathrm{II})$ oligothienyl complexes [27], a more direct general and highly effective "one-pot" synthesis of cyclo[ $n]$ thiophenes was developed. By using linear pentameric quinquethiophene $\mathbf{L 5 T}$ as a building block, a series of individual macrocycles $\mathrm{C} n \mathrm{~T}$, from C10T to an unprecedented size up to C35T, was obtained in an excellent overall yield of around $60 \%$. For the first time, CnTs including members with an odd number of repeating units became available on a preparative scale. Thus, the following macrocycles were isolated and characterized: C10T, C15T, C20T, C25T, C30T, and C35T [28]. X-ray structural analysis of C10T and characterization of its charged states revealed an unusual polaron pair structure of doubly oxidized $\mathbf{C 1 0 T}^{\mathbf{2}}$ serving as a model for charged and conducting states in linear oligo- and polythiophenes [29].

In this article, we present and analyze optical data on two larger series of cyclo $[n]$ thiophenes $\mathrm{C} n \mathrm{~T}$, series I and series II, which differ in ring size and substitution pattern of the solubilizing butyl side chains. The obtained structure-property relationships were further analyzed by our theoretical model based on Frenkel exciton theory. The comparison of the experimental with the theoretical spectral parameters gave valuable insights into the electronic structure, because they correlate the monomer transition energy $\left(\omega_{0}\right)$, the magnitude of the electronic coupling between the thiophene monomers in the macromolecules $(\mathrm{J})$, and the extent of the delocalized $\pi$-conjugated system [17].

\section{Experimental}

The solutions were freshly prepared with chloroform (Merck, UVASOL). UV-vis absorbance spectra were recorded on a Perkin-Elmer Lambda 19 spectrometer, and corrected fluorescence spectra were recorded on a Perkin-Elmer LS 55 under ambient conditions.

\section{Results and Discussion}

The macrocycles examined in this study are depicted in Scheme 1.

The first family of compounds (series I) is represented by alternately substituted thiophene units, every second unit having two butyl side chains in the $\beta$-positions leading to highly symmetrical derivatives: C8T, C12T, C16T, and C18T. Due to the
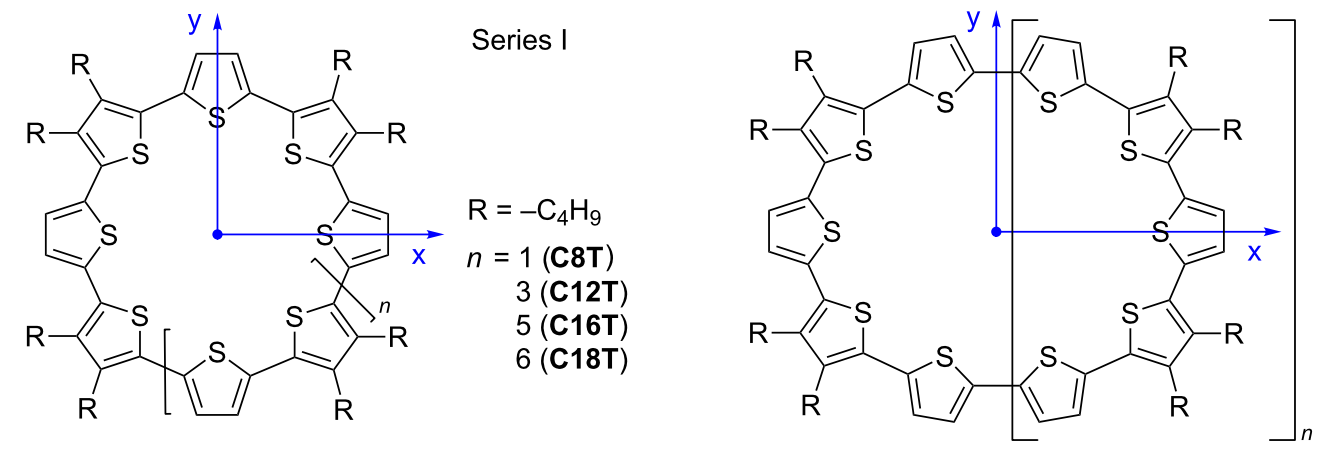

Series II
\[ \begin{aligned} \mathrm{R}= & -\mathrm{C}_{4} \mathrm{H}_{9} \\ n= & 1(\mathrm{C} 10 \mathrm{~T}) \\ & 2(\mathrm{C} 15 \mathrm{~T}) \\ & 3(\mathrm{C} 20 \mathrm{~T}) \\ & 4(\mathrm{C} 25 \mathrm{~T}) \\ & 5(\mathrm{C} 30 \mathrm{~T}) \\ & 6(\mathrm{C} 35 \mathrm{~T})\end{aligned} \]

Scheme 1: Chemical structure of the oligothiophene macrocycles (series I and II). The coordinate system used in the theoretical description is included. 
pentameric starting material L5T with dibutyl substitution at thiophenes 2 and 4, in series II a different alkyl chain substitution pattern resulted and allowed synthesis of macrocycles composed of an even or odd number of thiophene rings. Therefore, a much more extended series from C10T (cyclodimer) as the smallest member to $\mathbf{C 3 5 T}$ (cycloheptamer) as the largest was obtained.

The photophysical properties of the two series of macrocycles were analyzed by absorption and fluorescence spectroscopy. The data are summarized in Table 1. Two linear oligothiophenes, L5T and L10T, are included in the table as references having an identical substitution pattern to the macrocycles in series II. L5T is the building block used for the cyclization reactions and L10T the linear dimer.

Absorption spectra of the macrocycles in dichloromethane (DCM) at room temperature were characterized by several broad unstructured bands in the UV-vis region (Figure 1 and Figure 2).

In each series, the position of the low energy band, related to the $\pi-\pi^{*}$ transition of the macrocyclic systems, shifts on going from the smaller cycles to the larger ones. The absorption band

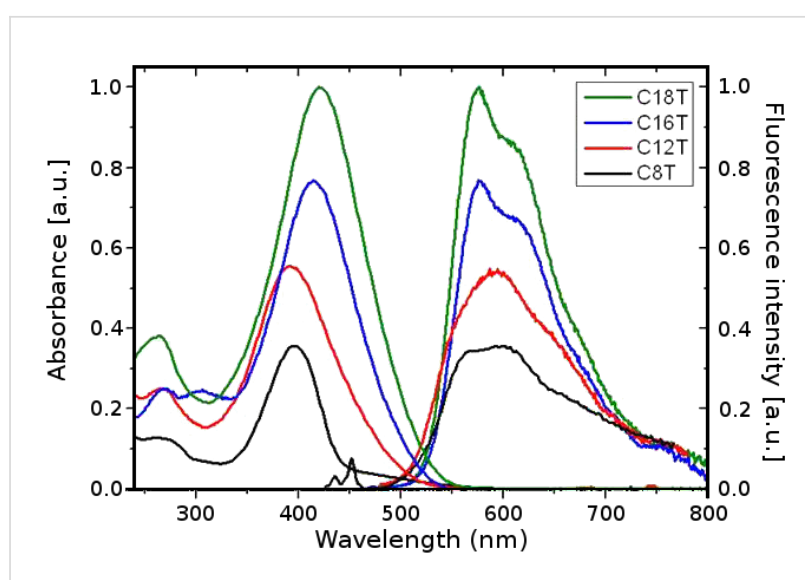

Figure 1: Absorption and fluorescence spectra of the macrocycles of Series I in dichloromethane (the excitation wavelength was chosen at the maximum of the absorption band).

( $\lambda_{\max }$ in Table 1) shifts to higher wavelengths with increasing number of thiophene units in the macrocycle $(\Delta \lambda=25 \mathrm{~nm}$ and $\Delta \lambda=28 \mathrm{~nm}$ in series I and II, respectively). The corresponding transition dipole lies in the molecular plane and is oriented almost parallel to the $\pi$-conjugated backbone, strongly dependent on the number of thiophene units composing the delocalized $\pi$-system, as in the case of the linear analogues [12].

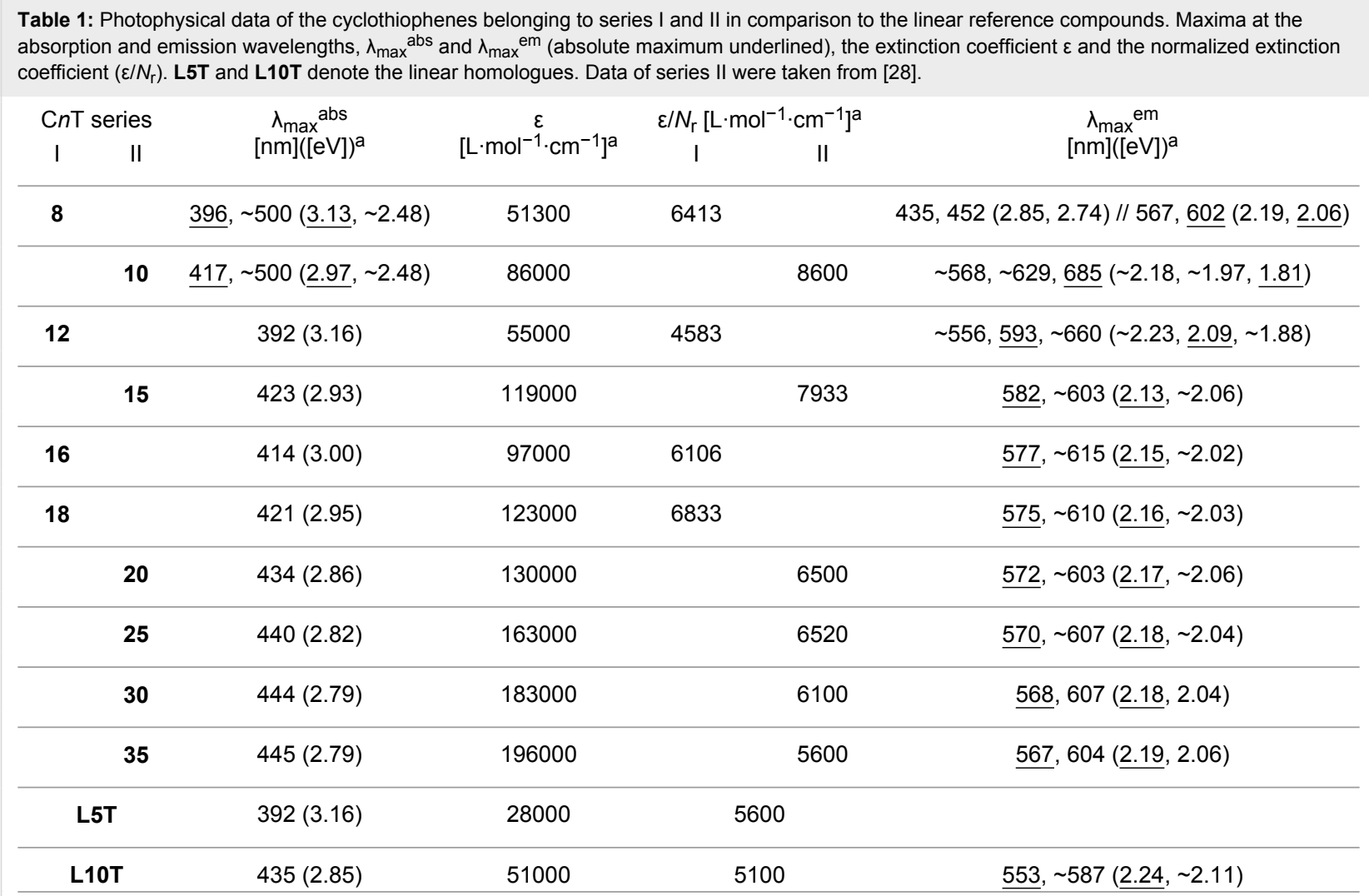

aSolvent: Dichloromethane. 


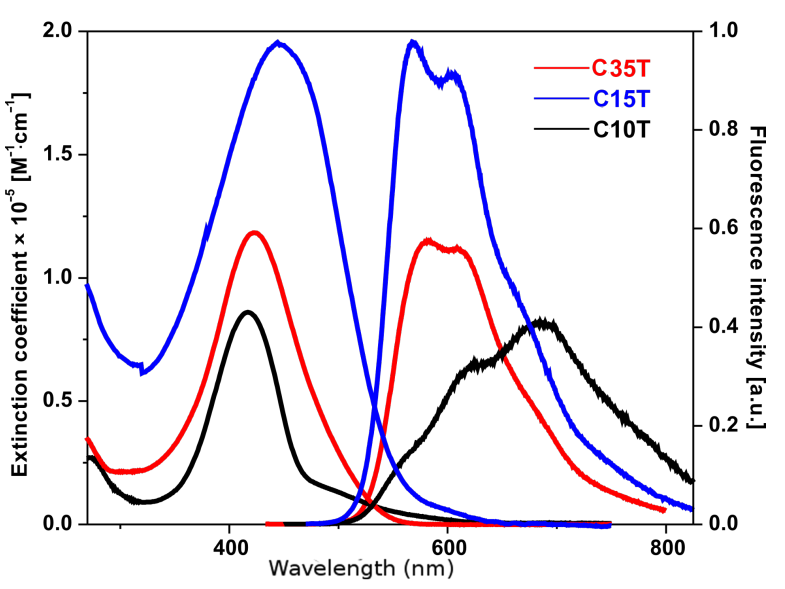

Figure 2: Representative absorption and fluorescence spectra of the smallest and largest macrocycles of series II in dichloromethane (taken from [28]). The excitation wavelength was chosen at the maximum of the absorption band.

Although a direct cross-correlation between the absorption of the two series seems inappropriate, the general trend shows that the macrocycles in series I absorb at higher energies than those of series II: C15T $\left(\lambda_{\max }=423 \mathrm{~nm}\right)$ absorbs at a higher wavelength compared to the next higher macrocycle C16T $\left(\lambda_{\max }=414 \mathrm{~nm}\right)$ : The blue-shift of the absorption band in series I indicates slightly lower $\pi$-conjugation due to bigger distorsions of the thiophenes induced by steric interactions of the increased number of alkyl chains ( 8 versus 6 for $\mathbf{C 1 6 T}$ and C15T, respectively).

A closer look at the smallest macrocycles (C8T and C10T) reveals a weak, but clear absorption band at lower energies $(\sim 500 \mathrm{~nm})$, which in the case of the bigger macrocycles is overlapped and covered by the stronger main absorption band
(Figure 1 and Figure 2). Because the selection rules for the electronic transitions of cyclic molecules substantially differ from those of the linear homologues, the transition to the lowest excited state in the macrocycles, which appears at $\sim 2.48 \mathrm{eV}$ independently of the ring size, is not allowed, whereas the degenerate second transition (corresponding to the intense absorption band) is permitted and ring-size dependent (see above) [12]. Figure 3 shows a sketch of the possible electronic transitions depending on the macrocycle size and taking into account the relative energies of the involved ground and excited states. The lack of vibronic structure in the absorption bands of all macrocycles indicates a nonplanar delocalized $\pi$-system with more or less well-pronounced torsion angles between the thiophene rings. This is in accordance with the behavior of the linear oligomers, which as well show a more pronounced aromatic structure in their ground state.

In both series the extinction coefficient increases with increasing macrocyclic ring size, although the values for series I are smaller than for series II (Table 1 and Figure 4, also see below Equation 6 and Equation 7). This effect can be attributed to the already mentioned distinct substitution pattern for both series. The extinction coefficient versus number of thiophenes in the cycle for series II fits well to a linear trend (red curve in Figure 4) with a slope of $4428 \mathrm{~L} \cdot \mathrm{mol}^{-1} \cdot \mathrm{cm}^{-1}$ per thiophene. This value is much lower than the normalized extinction coefficients ( $\varepsilon$ value $/ N_{\mathrm{r}}$ the number of thiophenes in the macrocycle) in this series, which decay linearly with the macrocycle size (Table 1) reaching the normalized value for the linear oligomers L5T and L10T $\left(\sim 5100 \mathrm{~L} \cdot \mathrm{mol}^{-1} \cdot \mathrm{cm}^{-1}\right)$. Due to this discrepancy, a better fit can be calculated taking the last value as a supplementary point into account: A second linear fit can be calculated for the smaller macrocycles (up to C15T) with a slope of $8058 \mathrm{~L} \cdot \mathrm{mol}^{-1} \cdot \mathrm{cm}^{-1}$. Interestingly, the normalized extinction

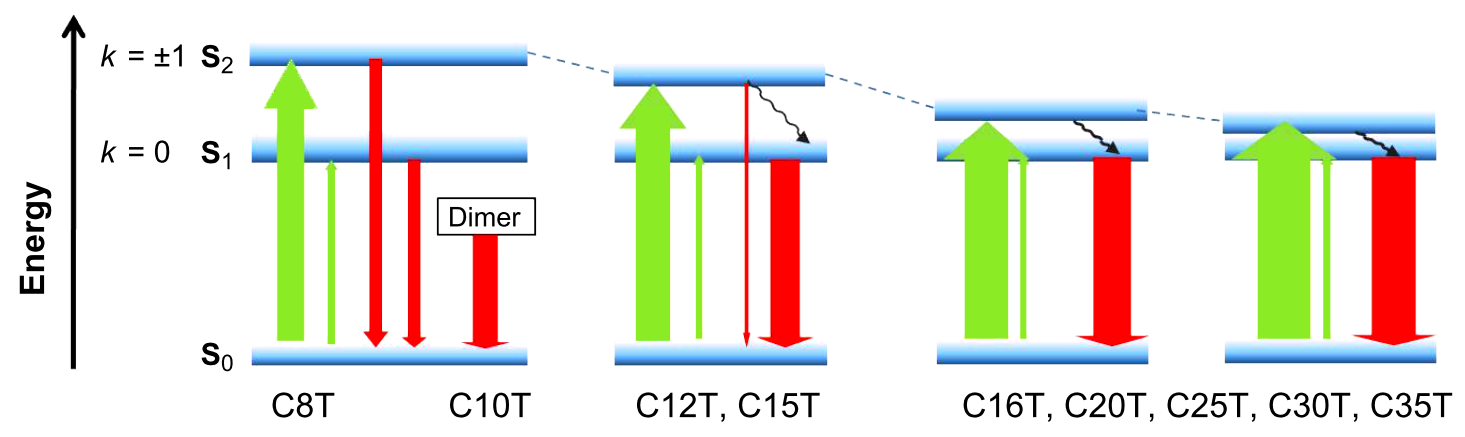

Figure 3: Sketch of the electronic transitions in the macrocycles: Ground state $\left(\mathrm{S}_{0}\right)$, first $\left(\mathrm{S}_{1}\right)$ and second $\left(\mathrm{S}_{2}\right)$ excited state. Plain arrows are weighted by the transition probability. The black wavy arrows represent nonradiative deactivation. 
coefficient remains almost constant in series I (exception C12T) with a value of about $6500 \mathrm{~L} \cdot \mathrm{mol}^{-1} \cdot \mathrm{cm}^{-1}$.

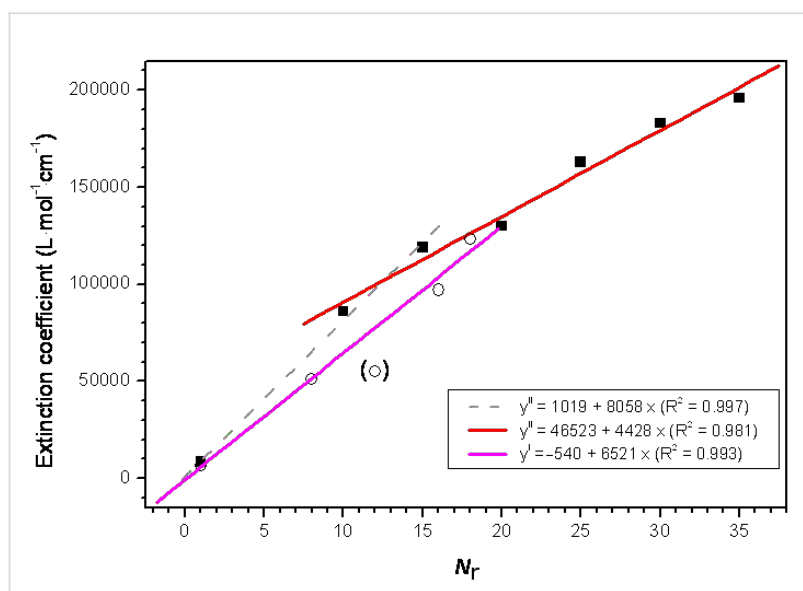

Figure 4: Extinction coefficient for the macrocycles of series I (circles) and II (squares) versus the number of thiophenes including the corresponding least-squares fit $\left(y^{\prime}\right.$ and $\left.y^{\prime \prime}\right)$ for the fit function $\varepsilon=a+b N_{r}$. Compound C12T (circle in parentheses) was not included in the linear fit of series $I$.

The fluorescence spectra revealed a structured emission band, in which up to four vibronic contributions can be observed. In contrast to the discussed torsion of thiophene units in the ground state of the macrocycles, we assume that in the excited state the macrocyclic conjugated backbone tends to planarize reducing the torsional angles of the thiophene units and in accordance to the linear analogues tends to a more quinoidal structure.

The fluorescence spectra of the larger macrocycles are alike with respect to their shape and energy position with maxima at around $2.15 \mathrm{eV}$ (series I) and $2.18 \mathrm{eV}$ (series II). For the small macrocycles, however, striking differences concerning the emission-band shape emerge and new bands appear, pointing to a special behavior most probably related to the inherent ring strain in these smaller homologues. The most relevant features concern the appearance of a very weak fluorescence band in the case of C8T at $\sim 450 \mathrm{~nm}$ and a more red-shifted emission in the case of C10T. The latter can be explained by the tendency of C10T to form dimers (excimers) in the excited state [29], which emit at lower energies with respect to the monomers $(1.81 \mathrm{eV}$ versus $2.18 \mathrm{eV}$ ). For the smallest cycle, C8T, we observe a weak structured emission band at much higher energy than the $\mathrm{S}_{0} \leftarrow \mathrm{S}_{1}$ transition, whose origin can be attributed to the emission from the second excited state, $\mathrm{S}_{2}$ (Figure 1, Table 1 and Figure 3). This double fluorescence behavior becomes possible because an incomplete energy transfer to the $S_{1}$ state occurs allowing partially the emission from the populated higher excited state $\mathrm{S}_{2}$.
In a previous theoretical work we showed that the optical excitations of thiophene-based linear and cyclic oligomers can be described by Frenkel excitons, which are delocalized over the oligomeric $\pi$-system $[12,30]$. Concerning the macrocycles of series I and II, we assume, as a first approach, that the electronic excitations of a thiophene subunit can be described by two relevant energy levels corresponding to the HOMO and the LUMO level. We assume that the energies of these states and the transition-matrix elements are essentially unmodified by the side chains. The Hamiltonian of the model then reads

$$
\begin{aligned}
H_{\mathrm{ex}} & =\omega_{0} \sum_{n=1}^{N_{\mathrm{r}}}|n\rangle\langle n| \\
& =-J \sum_{n=1}^{N_{\mathrm{r}}}(|n\rangle\langle n+1|+| n+1\rangle\langle n|),
\end{aligned}
$$

$\omega_{0}$ is the excitation energy, $N_{\mathrm{r}}$ the number of thiophene units in the macrocycle, $J$ describes the transfer of the excitation energy between neighboring thiophenes and $|n\rangle$ is the excited state at thiophene $n$. Throughout, we assume periodic boundary conditions, i.e., thiophenes 1 and $N_{\mathrm{r}}$ are nearest neighbors and complete the macrocyclic shape. The Schrödinger equation for this Hamiltonian can be represented as a difference equation. The energy eigenvalues and the eigenfunctions are given by the following expressions:

$$
\begin{gathered}
E_{k}=\omega_{0}-2 J \cos \left(\frac{2 \pi}{N_{\mathrm{r}}} k\right), \\
k=0, \pm 1, \pm 2, \ldots,\left\{\begin{array}{l} 
\pm\left(N_{\mathrm{r}}-1\right) / 2, N_{\mathrm{r}} \text { odd } \\
+N_{\mathrm{r}} / 2, N_{\mathrm{r}} \text { even }
\end{array}\right.
\end{gathered}
$$

The allowed optical transitions between the ground state $|0\rangle$ and the excited states (3) with energies given by (2) are determined by the matrix elements of the ring dipole operator:

$$
\vec{\mu}=\sum_{n=1}^{N_{\mathrm{r}}} \vec{\mu}_{n}(|0\rangle\langle n|+| n\rangle\langle 0|)
$$

Here $\vec{\mu}_{n}$ is the local optical dipole moment between the molecular ground and excited states. We assume that these dipole moments can have a component $\mu_{\|}$parallel to the z-axis and a component $\mu_{\perp}$ perpendicular to the z-axis, i.e., lying in the ring plane ( $\mathrm{x}-\mathrm{y}$ plane) and oriented in a tangential manner around the ring (Scheme 1) [31]. 
The evaluation of the z-component of the ring dipole moment $\mu_{\mathrm{z}}$ for the case that all molecular dipole moments $\mu_{\|}$are equal results in the following selection rule:

$$
\left|\left\langle 0\left|\mu_{\mathrm{z}}\right| k\right\rangle\right|^{2}=\mu_{\|}^{2} N_{\mathrm{r}} \delta_{k, 0}
$$

For the evaluation of the $\mathrm{x}$-component of the ring dipole moment $\mu_{\mathrm{x}}$ we again assume that the molecular dipole moments have the same $\mu_{\perp}$. However, the in-plane component for each molecule is oriented parallel to the ring tangent and thus its orientation is shifted by $2 \pi / N_{\mathrm{r}}$ as compared to those of the neighboring molecules. The evaluation of the dipole moment operator gives in this case

$$
\left|\left\langle 0\left|\mu_{\mathrm{x}}\right| k\right\rangle\right|^{2}=\mu_{\perp}^{2} \frac{N_{\mathrm{r}}}{4}\left(\delta_{k,-1}+\delta_{k, 1}\right)
$$

According to Equation 2 the two selection rules (Equation 6 and Equation 7) result in two different absorption lines, which agrees with our experimental finding of two absorption bands, more clearly observed for the small macrocycles. The first transition given for the component of the dipole moment perpendicular to the $\mathrm{x}-\mathrm{y}$ plane has the selection rule $k=0$ and their energy reads $E_{k}=\omega_{0}-2 J$, independent of the macrocycle size. The line position given for the component of the dipole moment in the $\mathrm{x}-\mathrm{y}$ plane has the selection rule $k= \pm 1$ and the line position reads $E_{k}=\omega_{0}-2 J \cos \left(2 \pi / N_{\mathrm{r}}\right)$. In the fit, using a leastsquares procedure in the Maple software, we determine the parameters $\omega_{0}$ and $J$, related to the monomer transition energy $\left(\omega_{0}\right)$ and the coupling energy between thiophene units $(J)$. The results of the fit (in $\mathrm{eV}$ ) are shown in Figure 5. The values corresponding to the smallest macrocycles in both series (C8T and C10T) were not included in the fitting, because of their evident deviation from the general trend. The reason for this behavior might be the ring strain of the macrocycle and the torsion that the individual thiophene rings experience due to their small ring size. This guess is supported by geometry calculations for C8T and X-ray structure analysis for C10T [10,29].

The macrocycles show an energetic decrease of their line positions, i.e., an increase of their wavelengths, with increasing ring size, which can be successfully described by Equation 2 and was observed experimentally. The circles show the experimental line positions of the macrocycle series I, and the squares show those of series II. The parameters of the fit are $\omega_{0}{ }^{\mathrm{I}}=5.713 \mathrm{eV}$, and $J^{\mathrm{I}}=1.472 \mathrm{eV}$ (series I, excluding C8T), and $\omega_{0}{ }^{\mathrm{II}}=4.853 \mathrm{eV}$, and $J^{\mathrm{II}}=1.051 \mathrm{eV}$ (series II, excluding C10T). We must stress here that the parameters obtained for the fit to series I should be treated with care because of the low number of macrocycles in the series ( 3 elements). The differ-

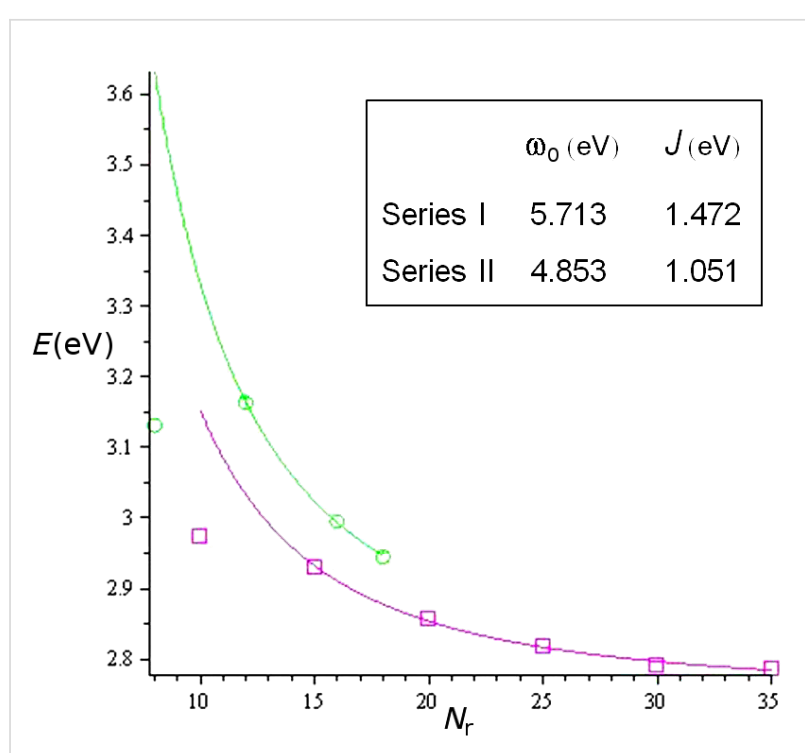

Figure 5: Diagram of the energy of the absorption band (eV) versus the number of thiophenes in the macrocycles of series I (circles) and II (squares), including the least-squares fit to Equation 2.

ence between the $\omega_{0}$ values can be attributed to the limitation of the theory, which does not take into account the thiophene substitution pattern. For comparison, the parameters obtained for the homologous linear oligomers are $\omega_{0}=5.5 \mathrm{eV}$ and $J=1.3 \mathrm{eV}$ [30]. Because of the strong sensitivity of the fit to the $\omega_{0}$ parameter, a better comparison can be performed by applying the well-established linear $\omega_{0}$ parameter to the two cyclic series, using it as a fixed value in the fitting and comparing the obtained coupling constants. These amount to $J^{\mathrm{I}}=1.355 \mathrm{eV}$ and $J^{\mathrm{II}}=1.388 \mathrm{eV}$ and reveal a slightly better electronic coupling for macrocycles of series II and therefore a reduced transition energy, which agrees with the experimentally red-shifted absorption of this series as compared to series I.

The experimentally observed linear increase of the extinction coefficients with increasing ring size (Figure 4) was also predicted by Equation 6 and Equation 7, confirming that the electronic excitation is distributed along the ring. These findings strongly corroborate the description of the electronic excitations by the model based on Frenkel excitons, despite the limitations assumed in this approach. A series of improvements of the model can be adopted in the future.

\section{Conclusion}

Oligothiophene macrocycles exhibit interesting photophysical properties. Due to the ring geometry, the electronic transitions follow distinct selection rules and the transition dipoles are mainly arranged in the macrocycle plane. Similar to the behaviour of linear oligomers, the absorption maxima positions 
tended towards a fixed value with increasing size of the macrocycle. The size-dependent effects on the absorption spectra (energy and extinction coefficient) can be described in the framework of a Frenkel exciton theory. The small macrocycles show most interesting photophysical behaviour (dual fluorescence), mostly due to their strong ring strain.

\section{Acknowledgements}

The authors gratefully acknowledge the financial support of the Deutsche Forschungsgemeinschaft within the collaborative research center SFB 569.

\section{References}

1. Fichou, D., Ed. Handbook of Oligo- and Polythiophenes; Wiley-VCH: Weinheim, Germany, 1999.

2. Bäuerle, P. Electronic Materials: The Oligomer Approach. Müllen, K.; Wegner, G., Eds.; Wiley-VCH: Weinheim, Germany, 1998; pp 105-197.

3. Roncali, J. Chem. Rev. 1997, 97, 173. doi:10.1021/cr950257t

4. Mishra, A.; Ma, C.-Q.; Bäuerle, P. Chem. Rev. 2009, 109, 1141. doi:10.1021/cr8004229

5. Kirschbaum, T.; Bäuerle, P. Synth. Met. 2001, 119, 127. doi:10.1016/S0379-6779(00)01206-6

6. Kirschbaum, T.; Azumi, R.; Mena-Osteritz, E.; Bäuerle, P. New J. Chem. 1999, 23, 241. doi:10.1039/A808026G

7. Mitschke, U.; Mena-Osteritz, E.; Debaerdemaeker, T.; Sokolowski, M.; Bäuerle, P. Chem.-Eur. J. 1998, 4, 2211. doi:10.1002/(SICI)1521-3765(19981102)4:11<2211::AID-CHEM2211> 3.3.CO;2-Z

8. Bäuerle, P.; Fischer, T.; Bidlingmeier, B.; Rabe, J. P.; Stabel, A. Angew. Chem., Int. Ed. 1995, 34, 303. doi:10.1002/anie.199503031

9. Krömer, J.; Rios-Carreras, I.; Fuhrmann, G.; Musch, C.; Wunderlin, M.; Debaerdemaeker, T.; Mena-Osteritz, E.; Bäuerle, P.

Angew. Chem., Int. Ed. 2000, 39, 3481. doi:10.1002/1521-3773(20001002)39:19<3481::AID-ANIE3481>3.0.CO ;2-O

10. Fuhrmann, G.; Debaerdemaeker, T.; Bäuerle, P. Chem. Commun. 2003, 948. doi:10.1039/b300542a

11. Fuhrmann, G. Synthesis and Characterization of Oligothiophene based Fully m-Conjugated Macrocycles. Ph.D. Thesis, University of Ulm, Ulm, Germany, 2006.

12. Zade, S. S.; Bendikov, M. J. Org. Chem. 2006, 71, 2972. doi:10.1021/jo0525229

13. Fomine, S.; Guadarrama, P. J. Phys. Chem. A 2006, 110, 10098 doi:10.1021/jp063065i

14. Fabian, J.; Hartmann, H. J. Phys. Org. Chem. 2007, $20,554$. doi:10.1002/poc.1203

15. Fomine, S.; Guadarrama, P.; Flores, P. J. Phys. Chem. A 2007, 111, 3124. doi:10.1021/jp0677750

16. Flores, P.; Guadarrama, P.; Ramos, E.; Fomine, S. J. Phys. Chem. A 2008, 112, 3996. doi:10.1021/jp710654k

17. Bednarz, M.; Reineker, P.; Mena-Osteritz, E.; Bäuerle, P. J. Lumin. 2004, 110, 225. doi:10.1016/j.jlumin.2004.08.013

18. Casado, J.; Hernández, V.; Ponce Ortiz, R.; Ruiz Delgado, M. C.; López Navarrete, J. T.; Fuhrmann, G.; Bäuerle, P. J. Raman Spectrosc. 2004, 35, 592. doi:10.1002/jrs.1202
19. Bhaskar, A.; Ramakrishna, G.; Hagedorn, K.; Varnavski, O.; Mena-Osteritz, E.; Bäuerle, P.; Goodson, T., III. J. Phys. Chem. B 2007, 111, 946. doi:10.1021/jp066766o

20. Varnavski, O.; Bäuerle, P.; Goodson, T., III. Opt. Lett. 2007, 32, 3083. doi:10.1364/OL.32.003083

21. Mena-Osteritz, E.; Bäuerle, P. Adv. Mater. 2001, 13, 243. doi:10.1002/1521-4095(200102)13:4<243::AID-ADMA243>3.0.CO;2-X

22. Mena-Osteritz, E. Adv. Mater. 2002, 14, 609. doi:10.1002/1521-4095(20020418)14:8<609::AID-ADMA609>3.0.CO;2 $-7$

23. Mena-Osteritz, E.; Bäuerle, P. Adv. Mater. 2006, 18, 447. doi:10.1002/adma.200501575

24. Mayor, M.; Didschies, C. Angew. Chem., Int. Ed. 2003, 42, 3176. doi:10.1002/anie.200250763

25. Jung, S.-H.; Pisula, W.; Rouhanipour, A.; Räder, H. J.; Jacob, J.; Müllen, K. Angew. Chem., Int. Ed. 2006, 45, 4685. doi:10.1002/anie.200601131

26. Nakao, K.; Nishimura, M.; Tamachi, T.; Kuwatani, Y.; Miyasaka, H.; Nishinaga, T.; Iyoda, M. J. Am. Chem. Soc. 2006, 128, 16740. doi:10.1021/ja067077t

27. Zhang, F.; Bäuerle, P. J. Am. Chem. Soc. 2007, 129, 3090. doi:10.1021/ja070083k

28. Zhang, F.; Götz, G.; Winkler, H. D. F.; Schalley, C. A.; Bäuerle, P. Angew. Chem., Int. Ed. 2009, 48, 6632. doi:10.1002/anie.200900101

29. Zhang, F.; Götz, G.; Mena-Osteritz, E.; Weil, M.; Sarkar, B.; Kaim, W.; Bäuerle, P. Chem. Sci. 2011, 2, 781. doi:10.1039/c0sc00560f

30. Bednarz, M.; Reineker, P.; Mena-Osteritz, E.; Bäuerle, P. Chem. Phys. 2007, 342, 191. doi:10.1016/j.chemphys.2007.09.052

31. Bednarz, M.; Knoester, J. J. Phys. Chem. B 2001, 105, 12913. doi:10.1021/jp012371n

\section{License and Terms}

This is an Open Access article under the terms of the Creative Commons Attribution License (http://creativecommons.org/licenses/by/2.0), which permits unrestricted use, distribution, and reproduction in any medium, provided the original work is properly cited.

The license is subject to the Beilstein Journal of Nanotechnology terms and conditions: (http://www.beilstein-journals.org/bjnano)

The definitive version of this article is the electronic one which can be found at: doi:10.3762/bjnano.2.78 